\title{
O uso de smartphones por Idosos durante a pandemia do COVID-19 no RN: um estudo exploratório.
}

\author{
Andressa Kroeff Pires, Isabel Dillmann Nunes \\ Instituto Metrópole Digital (IMD) - Universidade Federal do Rio Grande do Norte \\ (UFRN) - Av. Senador Salgado Filho, 3000, CEP 59.078.970 - Natal - RN - Brasil. \\ kroeffzinha@ufrn.edu.br, bel@imd.ufrn.br
}

\begin{abstract}
The world is facing the COVID-19 pandemic and measures of isolation and social distance have been adopted, mainly to protect the most vulnerable populations. This article identifies how elderly residents of $R N$ have used the smartphone during this pandemic and what are their main performed actions. The survey was answered by 26 individuals and includes demographic questions and smartphone-related usage. The results show that $76.9 \%$ of the elderly respect social isolation, 58\% learned something new with their smartphone and the subject of greatest interest for learning was to make purchases online.
\end{abstract}

Resumo: O mundo está enfrentando a pandemia do COVID-19 e medidas de isolamento e distanciamento social foram adotadas, principalmente para proteger populações mais vulneráveis. Este artigo identifica como idosos residentes do RN têm utilizado o smartphone durante essa pandemia e quais são as suas principais ações realizadas. A pesquisa foi respondida por 26 indivíduos e conta com questões demográficas e o uso de smartphones. Os resultados apontam que 76,9\% dos idosos respeitam o isolamento social, 58\% aprenderam algo novo com seu smartphone e o assunto de maior interesse para aprendizagem foi o de realizar compras online.

\section{Introdução}

Em 2012, a população brasileira atingiu o número de 25,4 milhões de idosos e, nos 5 anos seguintes, manteve a tendência de envelhecimento com um acréscimo de $18 \%$, ultrapassando os 30,2 milhões em 2017, sendo 56\%, a maioria, representada por mulheres (IBGE, 2018). Com base nos dados da SIDRA (2020), em 2019 o Nordeste brasileiro concentrou mais de 8 milhões de pessoas com 60 anos ou mais, e o estado do Rio Grande do Norte apresentou 523 mil idosos.

Essa tendência do envelhecimento da população brasileira, de acordo com Da Cruz, Caetano e Leite (2010), se dá devido às melhores condições de saúde que os avanços desta área proporcionam, resultando em mais qualidade de vida para as pessoas e, consequentemente, favorecendo o aumento da expectativa de vida. Mais recentemente, segundo informações do IBGE (2019), a expectativa de vida dos homens passou de 72,5 anos em 2017 para 72,8 anos em 2018, enquanto a das mulheres aumentou de 79,6 para 79,9 anos e a média brasileira ficou em 76,3 anos.

Neste ano de 2020, o mundo está enfrentando uma pandemia causada por um coronavírus, que provoca doenças respiratórias agudas, denominada COVID-19 
(NUNES, 2020). A população idosa é o grupo mais vulnerável que, ainda segundo Nunes (2020), pode ser acometido pela fase mais evoluída da doença, principalmente doenças crônicas, como patologias cardíacas, pulmonares, renais, diabetes, câncer, entre outras. Devido a pandemia, Hammerschmidt e Santana (2020) relatam que medidas de isolamento e distanciamento social foram adotadas, além de suspensão de aulas e trabalhos presenciais, para evitar a propagação rápida da doença.

Diante deste cenário, autores como Hammerschmidt e Santana (2020) levantam o questionamento sobre o estímulo de idosos a utilizar Tecnologias Digitais de Informação e Comunicação (TDICs), afirmando que essas permitem aproximação social. E Hayashi e Baranauskas (2017) complementam que o envelhecimento das pessoas em uma comunidade inclina-se a ampliar a distância delas da constante evolução das tecnologias digitais, o que dificulta o acesso e o uso das mesmas. Assim, Paschoal et al. (2016) destacaram a importância do uso das TDICs, pois estas podem ser integradas ao cotidiano, atendendo as necessidades e vivências das pessoas idosas, sendo para se entreter, se comunicar, trabalhar ou realizar atividades diárias com autonomia e mais qualidade de vida.

Em face disso, este artigo identifica como idosos, residentes do Rio Grande do Norte (RN), têm utilizado o smartphone durante a pandemia do COVID-19, quais são as principais ações realizadas com este dispositivo e quais são os assuntos que os respondentes têm interesse em aprender. A coleta de dados ocorreu através de um questionário criado no Google Formulários, onde os idosos responderam questões demográficas relacionadas ao isolamento social e sobre a utilização de smartphones.

Este trabalho organiza-se em quatro seções: a seção 2 apresenta os trabalhos relacionados ao escopo deste estudo; a 3a seção informa as etapas que compõem a metodologia. A seção 4 demonstra os resultados e discussão dos mesmos. Por fim, a 5a seção trata das considerações finais.

\section{Trabalhos relacionados}

Buscando trabalhos que se relacionam com a temática deste estudo, foi realizada uma pesquisa no portal da Comissão Especial de Informática na Educação (CEIE), por indexar sete diferentes revistas e anais de eventos voltados à Informática na Educação. Ao todo foram selecionados quatro artigos que tratam sobre a formação de idosos com relação ao uso de tecnologia e inclusão digital.

No artigo de Hayashi e Baranauskas (2017) foi desenvolvido um conteúdo programático de um curso sobre Letramento em Tecnologias Móveis para pessoas de meia e terceira idade com o intuito de aproximá-las aos meios de comunicação digitais. As pesquisadoras utilizaram a metodologia da Observação Participativa e Design Socialmente Consciente neste estudo, com isso foi aplicado o questionário Self Assessment Manikin (SAM) aos participantes com respostas afetivas (satisfação, motivação e domínio) sobre os assuntos abordados. Essas respostas foram bastante positivas, o que resultou na aprovação do conteúdo e metodologia do curso, o qual foi pensado com recursos e habilidades específicas para este grupo.

Recentemente, Guimarães, Ito e Yamanoe (2019) investigaram o impacto da Inclusão Digital na qualidade de vida das pessoas idosas, através da disciplina Inclusão 
Digital, do curso ofertado para este público específico. O curso iniciou com 38 alunos matriculados, com 28 alunos concluintes. Estes responderam um questionário com perguntas subjetivas sobre os assuntos aprendidos, sobre tecnologia e se, com o uso delas, houve cooperação na melhoria do bem-estar social e qualidade de vida. Os resultados foram positivos e apontaram que a Inclusão Digital contribuiu para que os idosos ganhassem maior independência, segurança e comodidade, além de mais cuidado com a saúde.

Oliveira, Fortes e Barbosa (2018) conduziram um estudo sobre o uso de dispositivos móveis e aplicações de aprendizagem móvel (m-learning app) com foco em usuários idosos, dividido em duas etapas: a aplicação de um questionário e entrevistas com especialistas em ensino para idosos. 28 pessoas participaram, mas 3 não tinham 60 anos ou mais. No questionário, foram feitas 19 questões que abordavam características gerais, uso de dispositivos móveis e interesse dos usuários em aplicações de aprendizagem móvel. As entrevistas tiveram mais 9 perguntas focadas em atividades que foram realizadas no curso de "Práticas de Tablets ou Smartphones para Terceira Idade". Os resultados evidenciaram a importância de uma aplicação mobile ser desenvolvida com características específicas, com destaque para a intuitividade. Como consequência, os pesquisadores criaram um protótipo de m-learning app, denominado Crossword Learning, com atividades do tipo palavras cruzadas para os idosos.

Foi apresentado o desenvolvimento de um aplicativo móvel, no estudo de Silva et al (2018), com ações que ajudam pessoas com Transtorno de Déficit de Atenção e Hiperatividade - TDAH e idosos com diagnóstico de Alzheimer, a se estimularem a aprender e exercitarem seu cérebro com o intuito de memorizar um novo conhecimento de forma eficaz, sem esquecer o anterior, e assim atenuar os efeitos da doença. Os pesquisadores usaram o Método de Memorização Exponencial Efetiva na Base Binária MMEEBB e Mapas de Conhecimento Estruturado - MCE para o processo de criação e desenvolvimento do aplicativo. Um grupo de 4 idosos com Alzheimer e 10 alunos com TDAH avaliaram algumas disciplinas de Computação. Os participantes que realizaram o método sugerido foram 3 idosos e 7 usuários com TDAH, que conseguiram manter o conhecimento adquirido recentemente na memória. E a média dos usuários nas disciplinas ficou de 70 a $90 \%$, constatando o aprendizado deles.

Esses trabalhos relacionados contribuem para este estudo por apresentarem metodologias e abordagens diferentes no que tange a temática aqui abordada. Em especial, o trabalho de Oliveira, Fortes e Barbosa (2018) se destaca por terem aplicado um questionário e realizado entrevistas com 28 idosos, em um curso de "Práticas de Tablets ou Smartphones para Terceira Idade", com o intuito de analisar o perfil dos aprendizes e propor um protótipo de m-learning app com atividades de palavras cruzadas. O relato de seus resultados alcançados e considerações finais contribuiu para a tomada de decisões neste artigo, auxiliando na etapa da análise dos dados coletados e na conclusão do mesmo.

\section{Metodologia}

Este artigo é um estudo exploratório e aplicado com abordagem quanti-quali, que seguiu um percurso metodológico de quatro etapas, a saber: (i) busca por trabalhos relacionados, como apresentada na Seção 2; (ii) elaboração de questionário; (iii) 
aplicação do questionário com o público alvo; e (iv) análise dos resultados obtidos, que ocorrerá na Seção 4, e escrita deste trabalho.

A ferramenta escolhida para criar o questionário foi o Google Formulários ${ }^{1}$, uma vez que esta permite a personalização do formulário - com vários tipos de campos -, armazena as respostas obtidas em uma planilha e gera gráficos para apresentar esses dados. Foram elaboradas 12 perguntas, que serão apresentadas na Tabela 1, de múltipla escolha, distribuídas em três etapas: (i) perfil pessoal; (ii) período de isolamento social; e (iii) uso de smartphone.

Tabela 1. Perguntas do questionário, organizadas por etapas.

\begin{tabular}{|c|c|c|}
\hline Etapa & Identificador & Questão \\
\hline \multirow[t]{4}{*}{ Perfil pessoal } & Q01 & Qual é o seu gênero? \\
\hline & Q02 & Qual é a sua faixa etária (idade)? \\
\hline & Q03 & Onde você mora? \\
\hline & Q04 & Qual a sua ocupação atual? \\
\hline \multirow{4}{*}{$\begin{array}{l}\text { Período de } \\
\text { isolamento } \\
\text { social }\end{array}$} & Q05 & Você recebe visitas em casa? \\
\hline & Q06 & Você tem saído de casa? \\
\hline & Q07 & Você mora sozinho? \\
\hline & Q08 & $\begin{array}{l}\text { Cite quais atividades você tem realizado quando sai de } \\
\text { casa? }\end{array}$ \\
\hline \multirow{4}{*}{$\begin{array}{l}\text { Uso de } \\
\text { smartphone }\end{array}$} & Q09 & Você tem smartphone? \\
\hline & Q10 & Você tem auxílio na utilização do celular? \\
\hline & Q11 & $\begin{array}{l}\text { Neste período de isolamento, você aprendeu a fazer } \\
\text { algo novo com seu smartphone? }\end{array}$ \\
\hline & Q12 & Sobre quais assuntos você gostaria de aprender? \\
\hline
\end{tabular}

Fonte: Elaboração própria.

A divulgação deste formulário foi realizada via mídias sociais: WhatsApp e Telegram, compartilhado em grupos com colegas e professores do programa de Pós-Graduação, pessoas do ambiente de trabalho destas pesquisadoras e grupos voltados para a área de Tecnologia da Informação (TI). Foi sugerido, na descrição do questionário, que alguma pessoa poderia auxiliar o idoso a preencher as questões, caso este não tivesse muita familiaridade com este tipo de ação. E o formulário ficou ativo por uma semana.

\footnotetext{
${ }^{1}$ https://www.google.com/intl/pt-BR/forms/about/
} 


\section{Resultados e discussão}

Conforme dito anteriormente, o questionário foi respondido por pessoas idosas, dos 27 participantes um foi desconsiderado pois reside no estado do Pernambuco. Na etapa denominada perfil pessoal, foram criadas perguntas sobre o gênero, faixa etária, cidade onde reside e ocupação atual. A maioria dos respondentes, 73\% (19), foi representada pelo gênero feminino e $27 \%$ (7), do masculino, totalizando 26 idosos respondentes. Em relação à idade, 65,4\% (17) se encontram na faixa etária de 60 a 69 anos, 23,1\% (6) entre 70 a 79 anos e apenas 11,5\% (3) participantes com 80 anos ou mais. Entre os respondentes, $88,5 \%$ (23) idosos residem em Natal, capital do RN, e os demais respondentes, 11,5\% (3), cada um mora em Parnamirim, Nísia Floresta e Mossoró. A maioria dos idosos, 76,9\% (20), são aposentados, 15,4\% (4) estão como servidores públicos e 7,7\% (2) pessoas informaram que sua ocupação é "do lar".

$\mathrm{Na}$ etapa sobre o período do isolamento social, 76,9\% (20) dos participantes responderam que não estavam recebendo visitas em casa, enquanto os $23,1 \%$ (6) restantes receberam visitas diariamente, semanalmente ou quinzenalmente, conforme mostra a Figura 1. Demonstrando que a maioria dos idosos estavam respeitando as normas de distanciamento social e se isolando em suas residências.

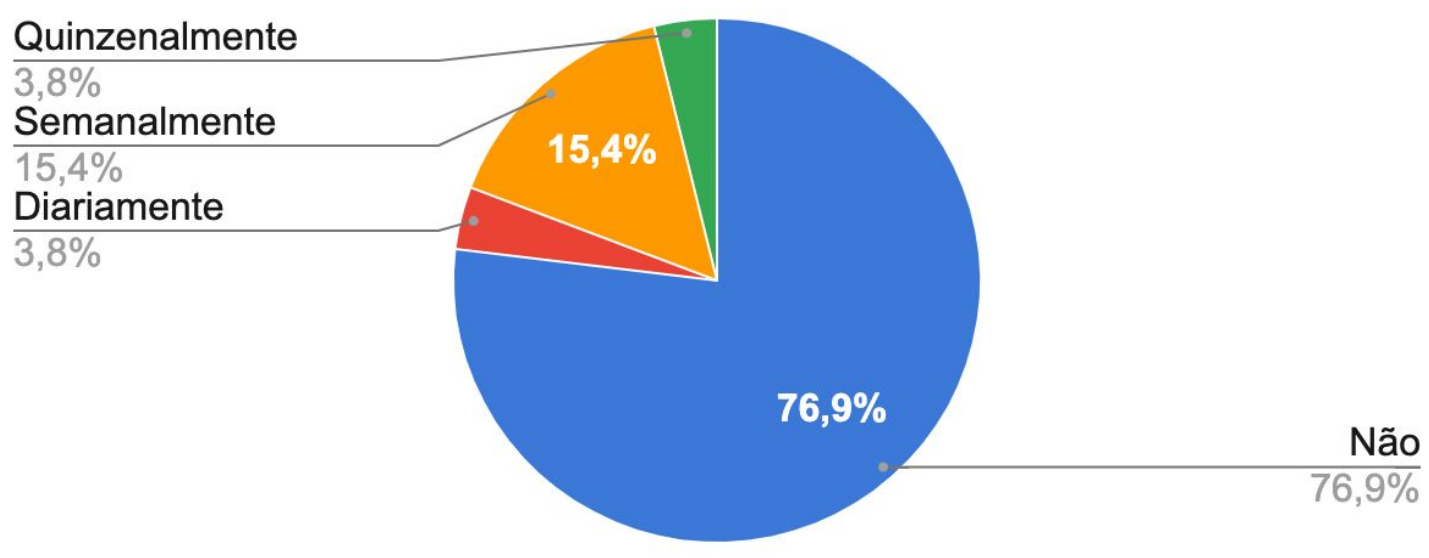

Figura 1. Porcentagem dos idosos que recebem, ou não, visitas em sua residência durante $o$ isolamento social. Elaboração própria.

Foi questionado, também, se os participantes estavam saindo de casa durante este período de pandemia, cerca de $81 \%$ (21) idosos responderam que não, e com isso passaram para a etapa seguinte. Já os demais respondentes, 19\% (5), tiveram que informar se moram sozinhos: $80 \%$ (4) responderam que sim e citaram quais atividades realizam quando saem de casa. Na Figura 2, apresentam-se as opções selecionadas ou informadas por eles. Percebe-se que as atividades externas a casa, são ações voltadas para a alimentação e saúde do idoso. O único idoso que respondeu que não mora sozinho foi redirecionado para a etapa seguinte. 


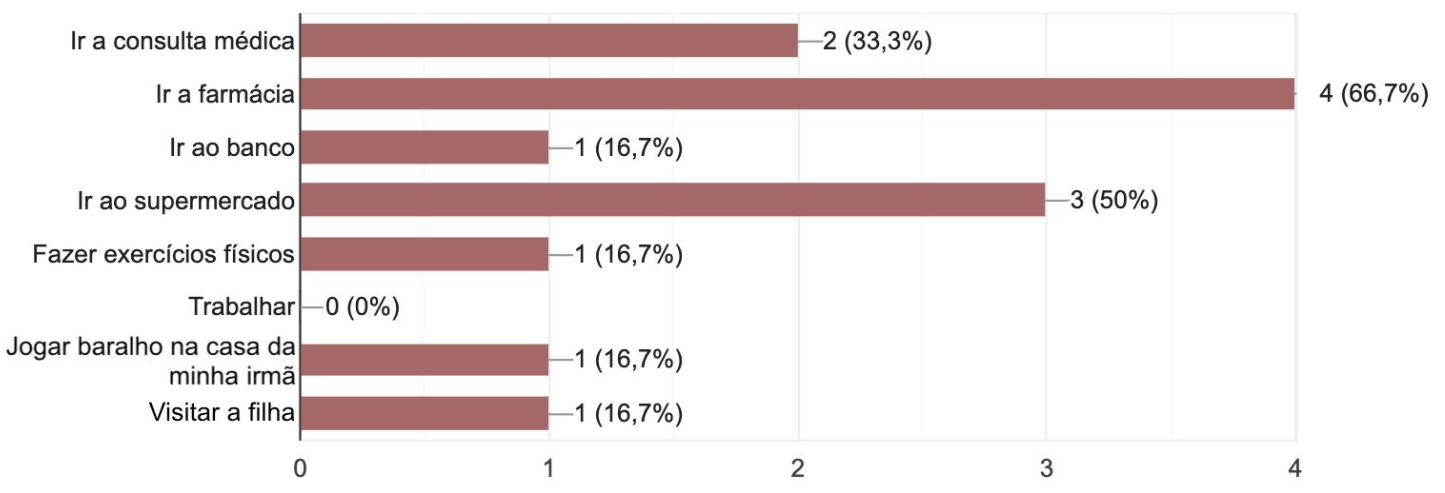

Figura 2. Atividades realizadas pelos idosos que estão saindo de casa durante o isolamento social. Elaboração própria.

Seguindo para as respostas sobre o uso de smartphones pelos idosos, apenas $7,69 \%$ (2) referiram não possuir este dispositivo móvel. Mais de 65\% (16) dos 24 respondentes têm auxílio para utilizar o celular. E neste período de isolamento, 53,85\% (14) idosos responderam que aprenderam a fazer algo novo com seu smartphone, a partir de opções selecionáveis, podendo escolher mais de um item e adicionar um novo. Na Tabela 2 serão listadas as opções pré-definidas, para auxiliar na análise dessa questão e da próxima.

Tabela 2. Opções sugeridas nas duas últimas questões do formulário.

\begin{tabular}{|l|l|}
\hline Identificador & Opção \\
\hline Op01 & Acessar a internet pelo navegador (sites) \\
\hline Op02 & Acessar o e-mail \\
\hline Op03 & Assistir vídeos no Youtube \\
\hline Op04 & Baixar um aplicativo / programa \\
\hline Op05 & Configurar o smartphone (tamanho de fonte, agenda, alarme, temas) \\
\hline Op06 & Fazer compras online \\
\hline Op07 & Jogar por um site ou aplicativo \\
\hline Op08 & Ouvir música \\
\hline Op09 & Participar de mídias sociais (Facebook, Instagram) \\
\hline Op10 & Realizar ações pelo aplicativo do banco \\
\hline Op11 & Usar aplicativos de comunicação (WhatsApp, Telegram) \\
\hline
\end{tabular}

Fonte: Elaboração própria.

As opções mais marcadas foram a Op09 com 7 votos, a Op04 e Op11, com 6 votos cada. E 28,6\% (4) dos participantes adicionaram um item cada, nesta pergunta em questão, os quais foram englobados em uma única ação identificada como "Participar de videoconferência / reunião (Google Meet, Zoom, Microsoft Teams)". Com base nessas 
ações, ficou evidente que estes idosos se interessaram pelas mídias sociais e meios de comunicação para manterem contato, mesmo que de forma virtual, com outras pessoas.

É importante ressaltar, também, que 75\% (3) dos idosos que informaram morar sozinhos, responderam que aprenderam algo novo no smartphone, a ação "Baixar um aplicativo/programa" obteve os votos de todos, em seguida com 2 votos foram "Acessar o e-mail (Gmail, Yahoo, Hotmail...)", "Realizar ações pelo aplicativo do banco" e "Participar de mídias sociais (Facebook, Instagram...)" e as demais tiveram apenas 1 voto cada, como pode ser visto na Figura 3.

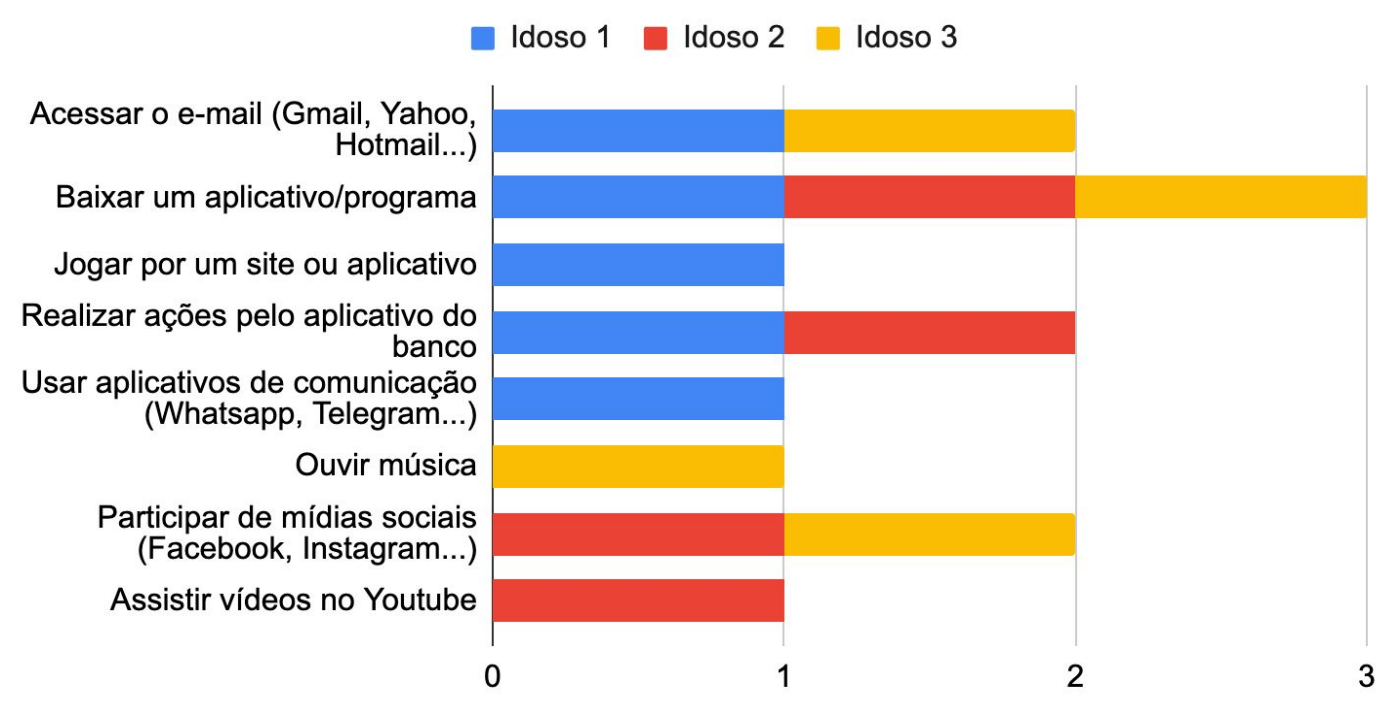

Figura 3. Ações aprendidas pelos idosos que moram sozinhos durante a pandemia. Elaboração própria.

Todas essas atividades, citadas anteriormente, podem contribuir com o desenvolvimento cognitivo do idoso, melhorando sua saúde mental e garantindo ganhos para a plasticidade cerebral (Guimarães, Ito e Yamanoe, 2019).

Por último, buscou-se identificar que assuntos os respondentes gostariam de aprender, relacionado ao uso dos smartphones, podendo selecionar as mesmas opções citadas na Tabela 2 e com possibilidade de adicionar assuntos do seu interesse. Segundo Guimarães, Ito e Yamanoe (2019), a terceira idade é uma geração que apresenta muita curiosidade e, como todas pessoas, independente de faixa etária, pode se dedicar a aprender o que deseja, se receber as condições mínimas para o processo de ensino-aprendizagem. 


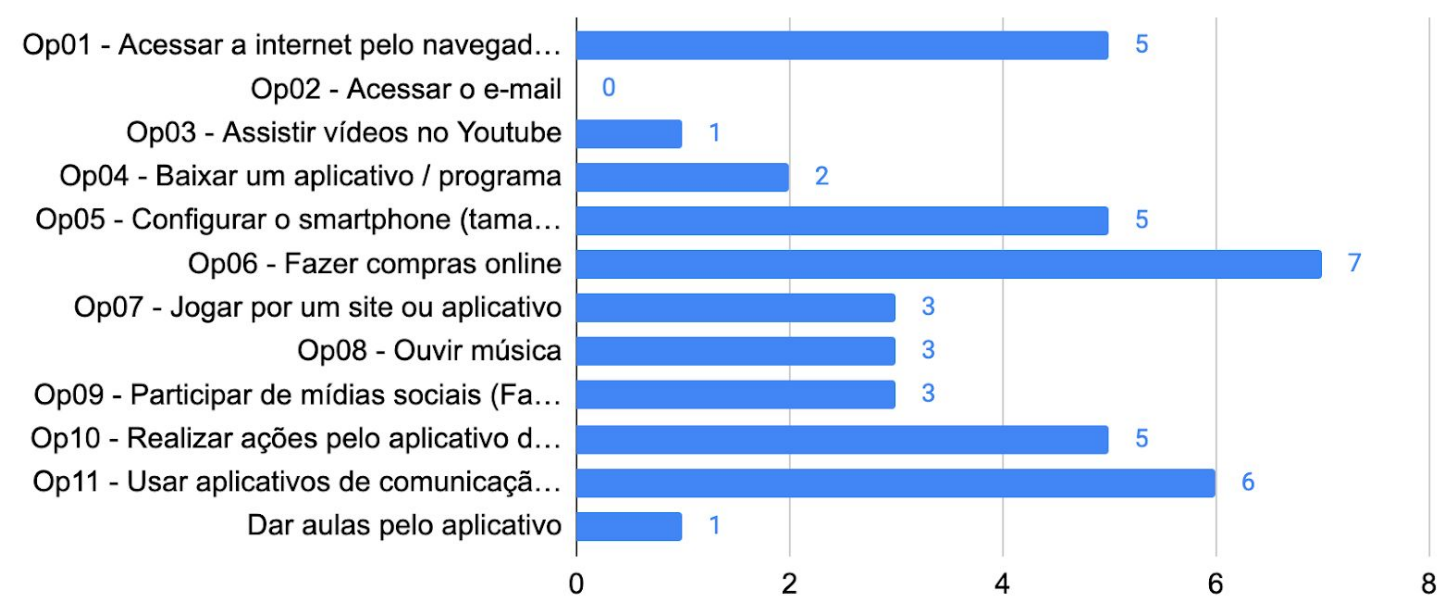

Figura 4. Assuntos que os idosos têm interesse em aprender. Elaboração própria.

Conforme apresenta a Figura 4, o assunto mais votado, com 7 respostas, foi "Fazer compras online", seguido de "Usar aplicativos de comunicação (WhatsApp, Telegram)" com 6 votos, e "Acessar a internet pelo navegador (sites)", "Configurar o smartphone (tamanho de fonte, agenda, alarme, temas)" e "Realizar ações pelo aplicativo do banco", todos com 5 votos. A única opção que não obteve votos foi "Acessar o e-mail", e uma idosa acrescentou o assunto "Dar aulas pelo aplicativo".

Esses números demonstram que os idosos estão buscando maior independência e autonomia com o auxílio e uso das tecnologias digitais, com destaque para os aplicativos de consumo, comunicação e bancários, facilitando a atividades cotidianas.

\section{Considerações finais}

Os dados apontam que a maioria dos idosos não mora sozinho e que estão realizando o isolamento e distanciamento social, para se proteger da pandemia do COVID-19. E o smartphone tem sido uma ferramenta importante que auxilia estes indivíduos no dia-a-dia, focando na comunicação com familiares, colegas de trabalho e conhecidos, assim como para o consumo e lazer. Mesmo neste momento difícil e atípico, os idosos estão conseguindo aprender a usar seus aparelhos celulares e a maioria demonstra interesse em aprender mais, com destaque para a realização de compras online e usar o aplicativo do banco.

Algumas dificuldades foram observadas, uma vez que 2 idosos aparentaram não compreender o que se pedia em nas questões Q03 e Q04, que estão identificadas na Tabela 1, e cujas respostas desses participantes específicos estão apresentadas na Tabela 3. Um outro idoso demonstrou, pelas suas respostas, não perceber que este formulário era específico para residentes no estado do Rio Grande do Norte, tendo sua participação sido desconsiderada para a seção de resultados e discussão deste artigo. 
Tabela 3. Respostas para as questões Q03 e Q04.

\begin{tabular}{|l|l|l|}
\hline Questão & Participante & Resposta \\
\hline Q3 & Idoso 1 & "PRAIA DE BUZIOS" \\
\hline Q4 & Idoso 2 & "Filmes na Netflix, livros de romances, etc" \\
\hline
\end{tabular}

Fonte: Elaboração própria.

Vale destacar, também, que o questionário ficou disponível para ser respondido por um período de 07 dias, pois esta pesquisa foi conduzida durante uma disciplina do curso de mestrado de uma das pesquisadoras, como parte integrante do levantamento de necessidades dos idosos atrelado ao seu projeto de pesquisa.

Por fim espera-se que trabalhos futuros possam ser realizados de forma a aprofundar o conhecimento sobre a aprendizagem dos idosos por smartphones, avaliando se o microlearning é eficaz. Este, juntamente com o micro-conteúdo (informações cujo tamanho é determinado por um único tópico, que abrange uma única idéia ou conceito) possibilitam o fornecimento de uma quantidade de conhecimento e informação, estruturada em vários capítulos curtos, refinados, bem definidos e interconectados (GIURGIU, 2017). Além de criar tutoriais com base nos assuntos mais votado por esses idosos e averiguar mais sobre a acessibilidade e usabilidade que uma aplicação precisa ter para atender da melhor forma a pessoa idosa.

\section{Referências}

DA CRUZ, D. T.; CAETANO, V. C.; LEITE, I. C. G. Envelhecimento populacional e bases legais da atenção à saúde do idoso. Cad. Saúde Colet., Rio de Janeiro, 18 (4): 500-8. 2010.

GIURGIU, L. Microlearning an evolving elearning trend. Scientific Bulletin, v. 22, n. 1. 2017.

GUIMARÃES, F.; ITO, G.; YAMANOE, M. C. P. Inclusão Digital na Terceira Idade: Considerações sobre a Experiência com a Informática. Anais do Workshop de Informática na Escola, v. 25, n. 1, p. 964, 11 nov. 2019.

HAMMERSCHMIDT, K. S. de A.; SANTANA, R. F. SAÚDE DO IDOSO EM TEMPOS DE PANDEMIA COVID-19. Cogitare Enfermagem, v. 25, n. 0, 28 abr. 2020.

HAYASHI, E.; BARANAUSKAS, M. C. Diminuindo a distância entre o envelhecer e a tecnologia móvel: uma proposta de curso. Anais do Workshop de Informática na Escola, v. 23, n. 1, p. 945, 27 out. 2017.

IBGE, AGÊNCIA DE NOTÍCIAS. Número de idosos cresce $18 \%$ em 5 anos e ultrapassa 30 milhões em 2017. Disponível em: https://agenciadenoticias.ibge.gov.br/agencia-noticias/2012-agencia-de-noticias/notic ias/20980-numero-de-idosos-cresce-18-em-5-anos-e-ultrapassa-30-milhoes-em-2017. Acesso em: 15 jul. 2020. 
IBGE, AGÊNCIA DE NOTÍCIAS. Em 2018, expectativa de vida era de 76,3 anos. Disponível em: https://agenciadenoticias.ibge.gov.br/agencia-sala-de-imprensa/2013-agencia-de-noti cias/releases/26104-em-2018-expectativa-de-vida-era-de-76-3-anos. Acesso em: 17 jul. 2020.

NUNES, V. M. de A. (ORG ). COVID-19 e o cuidado de idosos: recomendações para instituições de longa permanência. [s.1.] EDUFRN, 2020.

OLIVEIRA, C. de; FORTES, R.; BARBOSA, E. F. Um estudo sobre o uso de dispositivos móveis e aplicações de aprendizagem móvel com foco em usuários idosos. Brazilian Symposium on Computers in Education (Simpósio Brasileiro de Informática na Educação - SBIE), v. 29, n. 1, p. 1133, 28 out. 2018.

PASCHOAL, L. N.; LOPES, V.; CASSENOTE, M. R. S.; QUARESMA, C. R. T.; CHICON, P. M. M. Avaliação de um Jogo Sério digital destinado ao público idoso utilizando o método Gameflow. RENOTE, v. 14, n. 1, 26 jul. 2016.

SIDRA, SISTEMA IBGE DE RECUPERAÇÃO AUTOMÁTICA. Pesquisa Nacional por Amostra de Domicílios Contínua anual, Tabela 6407 - População residente, por sexo e grupos de idade. Disponível em: https://sidra.ibge.gov.br/tabela/6407\#/n1/all/n2/2/n3/24/v/606/p/last\%201/c2/all/c58/ 3302,95253/l/v,p+c2,t+c58/resultado. Acesso em: 17 jul. 2020.

SILVA, M.; SILVA, M.; DE MELO, S.; LIMA, T. O.; SOUZA, S.; LIMA, L. Uso dos MCE, MMEEBB e tecnologia computacional no processo de resgate e perenização do conhecimento a idosos e pessoas com atrasos de aprendizado. Brazilian Symposium on Computers in Education (Simpósio Brasileiro de Informática na Educação - SBIE), v. 29, n. 1, p. 983, 28 out. 2018. 